\title{
AN OPTIMISTIC FAIR EXCHANGE PROTOCOL FOR TRADING ELECTRONIC RIGHTS
}

\author{
Masayuki Terada \\ Network Management Development Dept., NTT DoCoMo \\ te@ rex.yrp.nttdocomo.co.jp
}

Makoto Iguchi

Information Sharing Platform Labs., NTT

iguchi@isl.ntt.co.jp

Masayuki Hanadate

Information Sharing Platform Labs., NTT

hanadate@isl.ntt.co.jp

Ko Fujimura

Information Sharing Platform Labs., NTT

fujimura@isl.ntt.co.jp

\begin{abstract}
Reliable electronic commerce systems must offer fairness. In this paper, we propose a fair exchange protocol for trading electronic vouchers, which are the representation of rights to claim goods or services. This protocol enables two players to exchange vouchers stored in their smartcards fairly and efficiently. The players can exchange vouchers through a 4-round mutual communication protocol between their smartcards as long as the protocol is performed properly. If the protocol becomes unable to proceed due to misbehavior of the partner or for any other reason, the player that has fallen into the unfair condition (i.e. the player who sent its voucher but didn't receive the desired voucher) can recover fairness by performing a recovery protocol with a trusted third-party. Since the recovery protocol doesn't need the cooperation of the partner, fairness can be recovered without identifying or tracking the partner; one can trade vouchers securely even if the trading partner cannot be identified.
\end{abstract}




\section{Introduction}

Fairness is essential for realizing secure electronic commerce in which both consumers and merchants can participate with a sense of safety. From the consumers' viewpoint, payment should not be committed without receiving the merchandise purchased, while the merchants may not want to send the goods or services prior to being paid.

Both of the above requirements can be achieved easily at face-to-face transactions in real shops. However, they are difficult in electronic commerce because payments and the delivery of merchandise are rarely conducted simultaneously.

Once people are disadvantaged, they need to identify the trading partner and acquire compensation. Unfortunately, identifying the partner is not easy in the Internet[4]. Since this difficulty is likely to become even worse in the consumer-to-consumer (C2C) market that is forming, security that depends on an identification process is impractical.

In order to realize secure electronic commerce systems that dispense with identification, we focus on "electronic rights", irreproducible and transferable digital data representing the rights to claim services or goods[14]. Their real world equivalents are pieces of paper, i.e. tickets, coupons and vouchers. Following RFC3506[5], we refer to such data as (electronic) vouchers hereafter. Since electronic vouchers must be transferred among users and redeemed in an off-line environment like real tickets and coupons, we assume that they are stored and managed in tamper-resistant devices like smartcards to prevent illegal acts like reproduction and forgery.

A voucher can represent diverse types of rights. For instance, it can represent the right to claim goods like "one hamburger", or to claim services like "one night accommodation". Moreover, it can be used as it were money by representing the right to claim conversion into currency or a certain amount of some valuable item (e.g. gold); electronic money can be treated as a sort of voucher. The fair exchange of vouchers, therefore, enables us to realize not only secure barter trading but also secure purchase transactions[7].

Identification of the trading partner is not required in these transactions provided that the fairness of the exchange is guaranteed. The only thing they need to certify is the genuineness of the vouchers being exchanged. Transaction security, therefore, doesn't depend on the trustworthiness of the trading partner, but rather on that of the voucher's issuer. This simplifies the certification process because there would be many fewer voucher issuers than participants. Fair exchange of vouchers is thus the key component to realize fair and secure electronic commerce while dispensing with the need for identification[6].

Up to now, however, there has been no efficient method to exchange vouchers with fairness. A mediating (or active) trusted third-party (TTP) can exchange vouchers fairly, but this approach has drawbacks in terms of availability and 
scalability because the TTP has to be synchronously involved in every exchange, which creates potential bottlenecks[11]. On the other hand, a number of optimistic fair exchange protocols have been proposed in which the TTP participates only if errors occur during the exchange[1, 11, 8, 17]. These protocols are much more efficient and practical for exchanging digital signatures (aka contract signing) or payments and receipts, but they cannot be used to exchange vouchers because they fail to prevent the reproduction of vouchers.

In this paper, we propose a new protocol that enables the fair and effective exchange of electronic vouchers that represent rights. This protocol exchanges vouchers in an optimistic manner; the trading participants first try to exchange vouchers through mutual communication, and they activate the TTP to recover fairness if the exchange is suspended or interrupted and can not be continued. Since this protocol guarantees "strong fairness" in any exchange, fairness can be recovered without identifying or tracking the trading partner.

The rest of the paper is organized as follows: Section 2 states the definitions and requirements for representing vouchers and fairness in voucher trading. This section also describes issues of the legacy method that uses a mediating TTP and previous optimistic fair exchange protocols. Section 3 details the protocol proposed in this paper. Section 4 discusses the proposed protocol by reference to the requirements stated in Section 2.

\section{Preliminaries}

This section states the definition and requirements of electronic vouchers and fairness in voucher exchanges. In addition, this section discusses drawbacks of the previous approaches.

\section{$2.1 \quad$ Electronic voucher}

An electronic voucher is a digital representation of the right to claim services or goods. In this context, the right is created by the issuer, such as a supplier of merchandise or provider of services, as a promise to the right holder. According to RFC3506[5], a voucher is defined as follows:

Electronic voucher Let $I$ be a voucher issuer, $H$ be a voucher holder, and $P$ be the issuer's promise to the right holder. An electronic voucher is defined as the 3-tuple of $(I, P, H)$.

Similar to paper tickets and current money, vouchers should be transferable. The voucher holder $H$ can transfer the voucher $(I, P, H)$ to another participant $H^{\prime}$. This transfer is represented as the rewriting of the tuple $(I, P, H) \rightarrow$ $\left(I, P, H^{\prime}\right)$. The right lapses from holder $H$ as a result of the transfer.

Vouchers must be protected from illegal acts like forgery, alteration, and reproduction. These security requirements are defined as follows: 
Preventing forgery A voucher $(I, P, H)$ must be generated (issued) only by issuer $I$ and must not be generated by any other participant ${ }^{1}$. In addition, it must not be possible for anyone to alter issuer $I$ once the voucher is issued.

Preventing alteration Once a voucher $(I, P, H)$ is issued, it must not be possible for anyone to alter promise $P$.

Preventing reproduction It must not be possible for voucher $(I, P, H)$ to be reproduced. In particular, in a transfer from $H$ to $H^{\prime}$, both $(I, P, H)$ and $\left(I, P, H^{\prime}\right)$ must not exist simultaneously throughout the transfer.

\subsection{Exchange of vouchers}

As mentioned in Section 1, diverse types of commerce transactions can be mapped into exchanges of vouchers. An exchange of vouchers consists of a pair of mutual transfers of vouchers.

To exchange vouchers fairly, it must be assured that no trading participant loses its voucher without receiving the desired voucher; each participant must be able to recover its voucher or the desired voucher in a given period as long as it behaves properly. An exchange of vouchers that satisfies this property is defined as a fair voucher exchange. Details are given below:

Fair voucher exchange Assume that there are two electronic vouchers $\left(I_{1}, P_{1}, H_{A}\right)$ and $\left(I_{2}, P_{2}, H_{B}\right)$. An exchange constructed of the pair of transfers $\left(I_{1}, P_{1}, H_{A}\right) \rightarrow$ $\left(I_{1}, P_{1}, H_{B}\right)$ and $\left(I_{2}, P_{2}, H_{B}\right) \rightarrow\left(I_{2}, P_{2}, H_{A}\right)$ is defined as a fair voucher exchange, if all of the following conditions are satisfied:

1 Provided that $H_{X}$ (i.e. $H_{A}$ or $H_{B}$ ) executes the exchange in proper manner, it is assured that $H_{X}$ will own either voucher as a result of the exchange. That is, either $\left(I_{1}, P_{1}, H_{X}\right)$ or $\left(I_{2}, P_{2}, H_{X}\right)$ is assured to exist at the termination of the exchange.

2 Provided that $H_{X}$ executes the exchange in proper manner, it is assured that $H_{X}$ can terminate the exchange in finite time.

The following conditions must also be satisfied to guarantee the prevention of voucher reproduction:

3 Throughout the exchange, $\left(I_{i}, P_{i}, H_{A}\right)$ and $\left(I_{i}, P_{i}, H_{B}\right)(i \in\{1,2\})$ must not exist simultaneously.

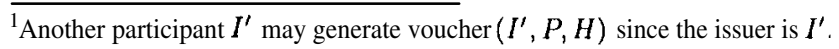




\subsection{Implementation model of vouchers}

Although several models can be used to implement electronic vouchers[6], we will focus on the "stored value" model like FlexToken[14]because this type of model has advantages in terms of usability and scalability.

This implementation model assumes that users (owners of vouchers) have tamper-resistant devices like smartcards to store and manage vouchers; it enables vouchers to be transferred among users and redeemed in off-line environments securely without involving banks or other third-parties. In this paper, we refer to these devices as PTDs (personal trusted devices). A PTD protects vouchers from illegal acts like forgery and reproduction from anyone, including its owner.

In this model, a voucher $(I, P, H)$ is considered to exist when user $H$ has PTD $U$ storing digital data $v$ that corresponds to $(I, P)$. In FlexToken, for example, PTDs store 2-tuple entries $(h(P k I), h(R))$, called a token for $v$, where $h()$ is a secure hash function like SHA-1 [10], $P k I$ is a public key of issuer $I$, and $R$ is a document called rights definition, which defines the contents of promise $P$.

The voucher transfer $\left(I, P, H_{A}\right) \rightarrow\left(I, P, H_{B}\right)$ is realized by transferring $v$ from sender's PTD $U_{A}$ to receiver's PTD $U_{B}$, which concludes by deleting $v$ from $U_{A}$ and storing $v$ in $U_{B}$.

\subsection{Previous works}

While the previous methods of implementing vouchers realize secure circulation of vouchers including issuance, transfer, and redemption with preventing illegal acts like forgery and reproduction, they don't realize fairness in the exchange of vouchers. Although FlexToken discusses fairness in circulating rights, it only aims to ensure the non-repudiation of the fact of circulation; ensuring fairness in voucher exchanges was not considered.

To achieve the fair exchange of vouchers, a voucher trading system that uses a mediating TTP has been introduced[7]. This system satisfies the fairness and security requirements described above, provided that the TTP acts honestly. However, it is weak in terms of availability because every exchange requires synchronous interactions between the TTP and both trading partners; its scalability is also suspect because traffic would be heavily focused on the TTP.

A number of fair exchange protocols for digital signatures and digital data have been proposed[1, 11, 8, 17]. In particular, protocols called optimistic protocols[2, 16, 15]or off-line TTP protocols[18]use a TTP only if errors occur in the exchange process. Under the assumption that errors rarely occur, these protocols relax the problems with using a TTP and enable exchanges to be performed efficiently. These protocols realize, in a practical manner, fair contract signings, certified mails, and fair exchanges of a payment and its receipt 
as demonstrated in SEMPER[9, 12], however, no optimistic protocol has been proposed or can be applied for exchanging vouchers.

In order to exchange vouchers fairly without identifying and tracing the trading partner, it is required to ensure fairness without an external dispute resolution process. This level of fairness is called strong fairness[11, 1].

According to [11], strong fairness can be achieved in an optimistic protocol if one of the items to be exchanged ensures "strong generatability" which means the item (or an equivalent item) is generatable by the TTP or "strong revocability" which means the item is revocable by the TTP 2 .

We first discuss protocols that use strong generatability and are based upon the protocol introduced in [2]. A voucher is strongly generatable if the TTP could generate a message equivalent to the message that ensures that a voucher is stored while preventing illegal acts on the voucher. However, a voucher is not strongly generatable in this sense because voucher reproduction is possible by replaying the first message of the protocol ${ }^{3}$. This message enables its recipient to perform the resolve protocol with the TTP, which may allow replay of the exchange ${ }^{4}$. This might be harmless for applications like contract signing because it only brings another evidence of confirmation of the same contract concluded in the previous exchange (assuming that contents of the contract are assured to be unique). However, it causes the recipient of the message to reproduce a voucher already received in the previous voucher exchange.

Applying a protocol that uses strong revocability[15]for voucher exchanges is possible but rather impractical. This protocol requires a means by which the TTP can ensure revocation that would prevent the receipt of improperly exchanged vouchers. This is easy for closed-loop electronic money systems (referred to "ECash-like system" in [15]) which involve a bank to confirm payments, but it is difficult for vouchers which have off-line capabilities similar to real tickets or current money; it is impractical to inform revocation to all participants who may receive the revoked voucher before the voucher is transferred or redeemed to them.

We therefore propose a new optimistic protocol for exchanging vouchers that is not based upon either type of protocol mentioned above. Our protocol prevents the replay attacks that make voucher reproduction possible. In addition, the protocol is simple and efficient; its main protocol consists of four messages, two of which are signed, and is as simple as a naive voucher exchange involving the mutual transfer of two vouchers using challenge and response.

\footnotetext{
${ }^{2}$ The other item is required to be "weakly generatable", which is rather easy to achieve.

${ }^{3}$ Another replay attack against this protocol is pointed out in [13], but it can be fixed easily as described in that paper.

${ }^{4}$ Additional message exchanges could prevent this replay attack, but it would make the whole protocol much more complicated.
} 


\section{Fair Exchange Protocol for Vouchers}

This section describes a fair exchange protocol that satisfies the requirements stated in Section 2.

In this section, we assume that the vouchers to be exchanged are $\left(I_{1}, P_{1}, H_{A}\right)$ and $\left(I_{2}, P_{2}, H_{B}\right)$, the implementation model is stored value model, $\left(I_{i}, P_{i}\right)$ is represented by token $v_{i}$, and voucher $\left(I_{i}, P_{i}, H_{X}\right)$ exists when $v_{i}$ is stored in PTD $U_{X}$ held by user $H_{X}$. The set of tokens stored in $U_{\boldsymbol{X}}$ is referred to as $V_{\boldsymbol{X}}$. PTD $U_{\boldsymbol{X}}$ is a tamper-resistant device like a smartcard which is capable of preventing illegal alteration of $V_{X}$ or the program performing the exchange, as well as keeping its signing key and $\boldsymbol{n}_{\mathbf{2}}$ (described in 3.2) secret. Each process in the PTD is assumed to be performed atomically.

User $\boldsymbol{H}_{\boldsymbol{X}}$ would also have a user terminal device such as a mobile phone or a PDA to interact with its PTD $U_{X}$ if the PTD doesn't have a user interface (like a smartcard). The user terminal would facilitate the generation of communication channels among PTDs and TTP $T$ (see below) as well, but it doesn't have to be trusted by anyone but its owner; the owner might try to cheat its PTD or another PTD by forging or replaying messages using the terminal. The terminals aren't shown explicitly hereafter since they can be merely treated as a part of (insecure) communication channels in the proposed protocol.

This protocol consists of three sub-protocols: a main protocol, an abort, and a resolve protocol. An exchange starts by performing the main protocol. The exchange completes only within the main protocol whenever both participants act honestly and there is no trouble in the communication channel between them. If there are any troubles in the main protocol, either participant can recover fairness and terminate the exchange with the abort protocol or the resolve protocol using TTP $T$, which is a third-party trusted by both participants (and also the issuers of the vouchers). This protocol exchanges vouchers fairly and optimistically.

\subsection{Definitions}

The other definitions and assumptions needed to describe the proposed protocol are given below (details are given in Section 4).

$U_{A}$ has public key certificate $\operatorname{Cert}_{A}$ including public key $P k A$, a signing key which generates a signed message $(m)_{P k A}$ verifiable with $\operatorname{Cert}_{A}$, and a verify function $\operatorname{Verify}\left((m)_{P k X}, \operatorname{Cert}_{X}\right)$ which verifies the signed message $(m)_{P k X}$ using the corresponding certificate $\operatorname{Cert}_{X}$; and $U_{B}$ and $T$ likewise.

$\operatorname{Cert}_{A}$ and $\operatorname{Cert}_{B}$ represent that their keyholders $U_{A}$ and $U_{B}$ are certified as proper PTDs, while $\operatorname{Cert}_{T}$ represents that its keyholder $T$ is certified as a proper TTP. Note that these certificates don't have to be identity certificates to identify individuals, but have to be issued by a party who is trusted by the issuer of the vouchers being exchanged. It is easy to ensure this requirement if the 


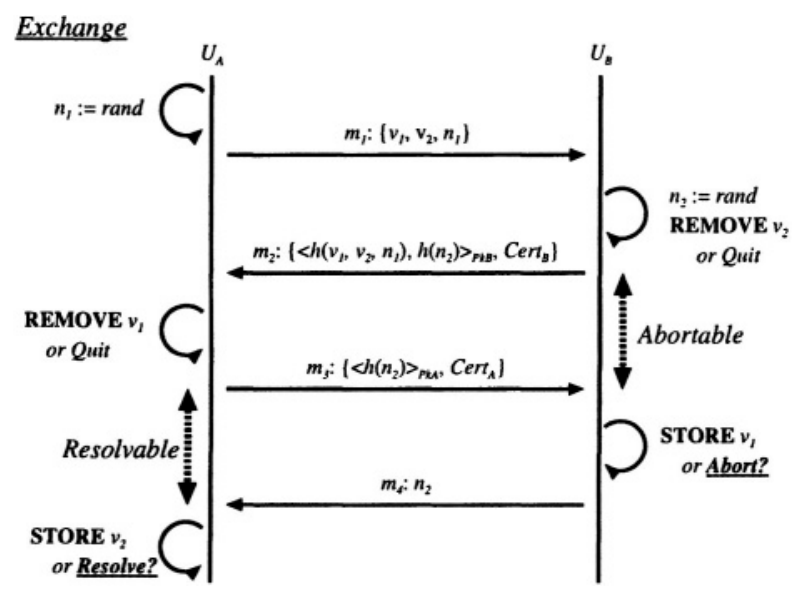

Figure 1. Main protocol.

existence of a certificate authority trusted by all participants can be assumed. If not, additional certificates by the issuer like accredit information in FlexToken can be used (see details in [14]).

In addition, $U_{A}$ and $U_{B}$ manage sets of sessions $S_{A}$ and $S_{B}$ respectively, and $T$ manages sets $S_{a b o r t}$ and $S_{\text {resolve }}$ which include sessions that have been aborted or resolved.

\subsection{Main protocol}

Figure 1 shows the main protocol. At the initial point, tokens $v_{1}$ and $v_{2}$ are stored in $U_{A}$ and $U_{B}$ respectively $\left(v_{1} \in V_{A}, v_{2} \in V_{B}\right)$. If this protocol terminates without failure, $v_{1}$ and $v_{2}$ are swapped $\left(v_{1} \in V_{B}, v_{2} \in V_{A}\right)$. The exchange is performed without intervention of the TTP in this case. In the explanation hereafter, it is assumed that $H_{A}$ knows the content of $v_{2}$ at the initial point for simplicity.

$H_{B}$ may order $U_{B}$ to abandon this protocol at any time in the abortable section in Figure 1 (after sending $m_{2}$ and before receiving $m_{3}$ ) and terminate the exchange by performing the abort protocol with $T$ which recovers fairness for $H_{B}$. Likewise, $H_{A}$ may order $U_{A}$ to terminate the exchange by performing the resolve protocol with $T$ throughout the resolvable section (after sending $m_{3}$ and before receiving $m_{4}$ ). Before these sections, $H_{A}$ and $H_{B}$ can merely quit the exchange without losing fairness.

$H_{A}$ starts the main protocol by ordering $U_{A}$ to exchange $v_{1}$ in $U_{A}$ and $v_{2}$ in $U_{B}$. The main protocol is performed in the following way: 
1 After receiving the order from $H_{A}, U_{A}$ performs the following:

(a) Generates a random number $n_{1}$ and adds it to set $S_{A}$.

(b) Sends $m_{1}:\left\{v_{1}, v_{2}, n_{1}\right\}$ to $U_{B}$, which is the offer of the exchange.

$2 U_{B}$ receives $m_{1}$ and performs the following:

(a) Confirms $m_{1}$ if it is an acceptable offer for $H_{B}$. If not, $U_{B}$ quits the exchange (and may inform $U_{A}$ of this event).

(b) Generates $n_{2}$ and adds it to set $S_{B} ; n_{2}$ has to be kept in secret until it is sent as $m_{\mathbf{4}}$.

(c) Removes $v_{2}$ from $V_{B}$, which causes the corresponding rights to lapse from $H_{B} ; H_{B}$ temporarily falls into unfair condition.

(d) Calculates $s_{1}:=h\left(v_{1}\left|v_{2}\right| n_{1}\right)$ and $s_{2}:=h\left(n_{2}\right)$.

(e) Sends $m_{2}:\left\{\left(s_{1} \mid s_{2}\right)_{P k B}, \operatorname{Cert}_{B}\right\}$ to $U_{A}$.

$3 U_{A}$ receives $m_{2}$ and performs the following:

(a) Confirms that all of the following equations are satisfied.

$$
\begin{aligned}
& \text { i } s_{1}=h\left(v_{1}\left|v_{2}\right| n_{1}\right) \\
& \text { ii Verify }\left(m_{2}\right)=\text { true }
\end{aligned}
$$

If not, $U_{A}$ waits $m_{2}$ again or quits the exchange by removing $n_{1}$ from $S_{A}$.

(b) Removes $n_{1}$ from $S_{A}$ and adds $\boldsymbol{s}_{\mathbf{2}}$ to $\boldsymbol{S}_{\boldsymbol{A}}$.

(c) Removes $v_{1}$ from $V_{A}$, which causes the corresponding rights to lapses from $H_{A} ; H_{A}$ temporarily falls into unfair condition (That is, $H_{A}$ and $H_{B}$ fall into unfair condition at this time).

(d) Sends $m_{3}:\left\{\left(s_{2}\right)_{P k A}, \operatorname{Cert}_{A}\right\}$ to $U_{B}$.

$4 U_{B}$ receives $m_{3}$ and performs the following:

(a) Confirms that all of the following equations are satisfied.

$$
\begin{aligned}
& \text { i } s_{2}=h\left(n_{2}\right) \\
& \text { ii } \operatorname{Verify}\left(m_{3}\right)=\text { true }
\end{aligned}
$$

If not, $U_{B}$ waits $m_{3}$ again or abandons the main protocol and performs the abort protocol to recover fairness.

(b) Removes $n_{2}$ from $S_{B}$ and adds $v_{1}$ included in $m_{1}$ to $V_{B} . H_{B}$ enters fair condition again.

(c) Sends $m_{4}: n_{2}$ to $U_{A}$, and $U_{B}$ terminates the exchange.

$5 U_{A}$ receives $m_{4}$ and performs the following: 


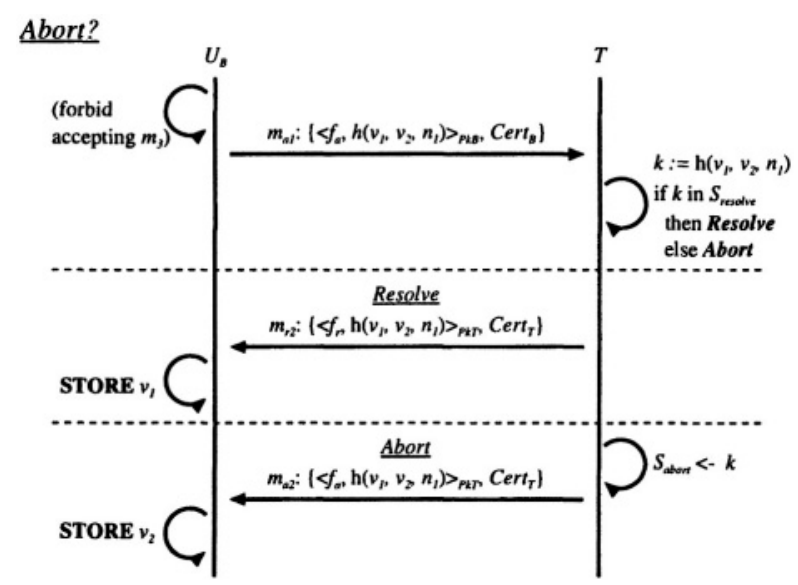

Figure 2. Abort protocol.

(a) Confirms $h\left(m_{4}\right)=s_{2}$. If not, $U_{A}$ waits $m_{4}$ again or performs the resolve protocol to recover fairness.

(b) Removes $s_{2}$ from $S_{A}$ and adds $v_{2}$ to $V_{A} . H_{A}$ enters fair condition again, and $U_{A}$ terminates the exchange.

\subsection{Abort protocol}

Figure 2 shows the flow of the abort protocol. As mentioned above, $U_{B}$ may abandon the main protocol at any time in the abortable section by performing this protocol.

The abort protocol enables $U_{B}$ to recover fairness by sending an abort request $m_{a 1}$ to $T$ and receiving the abort admission $m_{a 2}$ which allows $U_{B}$ to restore $v_{2}$, or the resolve admission $m_{r 2}$ to store $v_{1}$ if $T$ has already received the resolve request from $U_{A}$. If $U_{B}$ cannot receive either $m_{a 2}$ or $m_{r 2}$ from $T$ in a given period, $U_{B}$ may resend $m_{a 1}$.

The abort protocol is performed as follows:

$1 U_{B}$ abandons the main protocol, and is prohibited from receiving $m_{3}$ in the main protocol.

$2 U_{B}$ sends $m_{a 1}:\left\{\left(f_{a} \mid s_{1}\right)_{P k_{B}}\right.$, Cert $\left._{B}\right\}$ to $T$. Herein, $f_{a}$ is a flag which represents the process of aborting.

$3 T$ receives $\boldsymbol{m}_{\boldsymbol{a} \mathbf{1}}$ and performs the following:

(a) Confirms Verify $\left(m_{a 1}\right)$. If not, waits $m_{a 1}$ or $m_{r 1}$ (described in the resolve protocol) again. 
(b) Let $k:=s_{1}$,

i If $k \in S_{\text {resolve, then send the resolve admission. }}$

ii If $k \notin S_{\text {resolve }}$, then add $k$ to $S_{\text {abort }}$ and send the abort admission.

The procedures $U_{B}$ performs when $U_{B}$ receives the abort admission or the resolve admission are as follows:

\section{Receiving abort admission}

$1 T$ sends $m_{a 2}:\left\{\left(f_{a} \mid k\right)_{P k T}, \operatorname{Cert}_{T}\right\}$ to $U_{B}$.

$2 U_{B}$ receives $m_{a 2}$ and performs the following:

(a) Confirms $k=s_{1}$ and Verify $\left(m_{a 2}\right)$. If not, waits $m_{a 2}$ or $m_{r 2}$ again.

(b) Removes $\boldsymbol{n}_{2}$ from $\boldsymbol{S}_{\boldsymbol{B}}$.

(c) Adds $v_{\mathbf{2}}$ to $V_{\boldsymbol{B}}$ and terminates the exchange.

\section{Receiving the resolve admission}

$1 T$ sends $m_{r 2}:\left\{\left(f_{r} \mid k\right)_{P k T}\right.$, Cert $\left._{T}\right\}$ to $U_{B}$.

$2 U_{B}$ receives $m_{r 2}$ and performs the following:

(a) Confirms $k=s_{1}$ and $\operatorname{Verify}\left(m_{r 2}\right)$. If not, waits $m_{a 2}$ or $m_{r 2}$ again.

(b) Removes $n_{\mathbf{2}}$ from $S_{B}$.

(c) Adds $v_{1}$ to $V_{B}$ and terminates the exchange.

\subsection{Resolve protocol}

Figure 3 shows the flow of the resolve protocol. $U_{A}$ may perform this protocol at any time in the resolvable section of the main protocol.

Similar to the abort protocol, the resolve protocol enables $U_{\boldsymbol{A}}$ to recover fairness by sending a resolve request $m_{r 1}$ to $T$ and receiving the abort admission $m_{a 2}$ which allows $U_{A}$ to restore $v_{1}$, or the resolve admission $m_{r 2}$ to store $v_{2}$ if $T$ has already received the abort request from $U_{B}$. If $U_{A}$ cannot receive either $m_{a 2}$ or $m_{r 2}$ from $T$ in a given period, $U_{A}$ may resend $m_{r 1}$.

The resolve protocol is performed as follows:

$1 U_{A}$ sends $m_{r 1}:\left\{\left(f_{r} \mid s_{1}\right)_{P k A}, \operatorname{Cert}_{A}\right\}$ to $T$. Herein, $f_{r}$ is a flag that represents the process of resolving.

$2 T$ receives $\boldsymbol{m}_{r 1}$ and performs the following: 


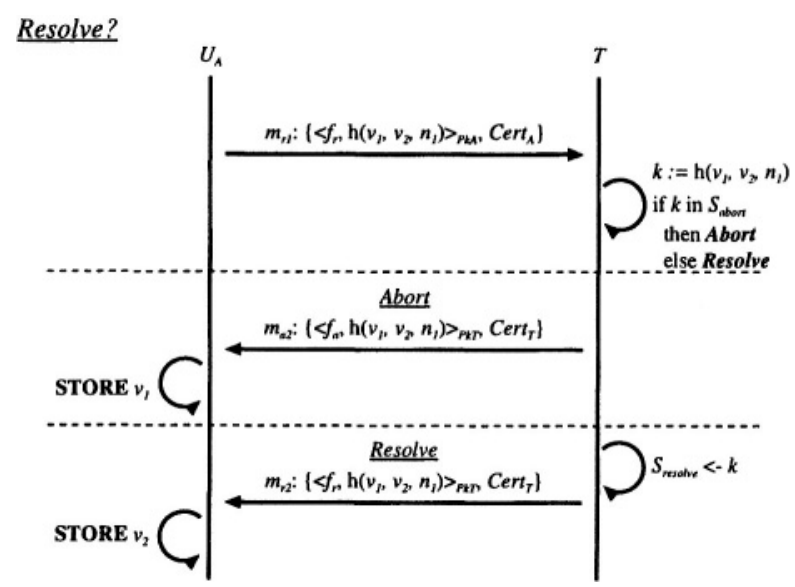

Figure 3. Resolve protocol.

(a) Confirms Verify $\left(m_{r 1}\right)$. If not, waits $m_{a 1}$ or $m_{r 1}$ again.

(b) Let $k:=s_{1}$,

i If $k \in S_{a b o r t}$, then send the abort admission,

ii If $k \notin S_{\text {abort }}$, then add $k$ to $S_{\text {resolve }}$ and send the resolve admission.

$U_{A}$ processes the abort admission or the resolve admission in a similar way to $U_{B}$ in the abort protocol, except for the following differences:

- $U_{B}$ is replaced by $U_{A}$.

- Deleting $n_{2}$ from $S_{B}$ is replaced by deleting $s_{2}$ from $S_{A}$.

- $v_{1}$ and $v_{2}$ stored by receiving $m_{r 2}$ or $m_{a 2}$ are swapped.

- $U_{A}$ doesn't have to abandon the main protocol. $U_{A}$ may conclude the exchange by receiving $m_{4}$ in the main protocol before receiving $m_{a 2}$ or $m_{r 2}$, because it can be assumed that $U_{B}$ has successfully concluded the exchange when $U_{A}$ receives $m_{4}$ and therefore there is no chance for $U_{A}$ to receive the abort admission $m_{a 2}$.

\section{Discussions about Security and Fairness}

This section discusses how the proposed protocol ensures the security requirements for vouchers and the fairness requirement.

In the discussion hereafter, the following assumptions are made: 
1 Users $H_{A}$ and $H_{B}$ may try to cheat their PTDs; they might replay or forge any messages.

2 Tamper-resistant capability of both PTDs, $U_{A}$ and $U_{B}$, is not compromised; they process the input data $m_{i}$ properly and their signing keys are kept secret. In addition, $\boldsymbol{n}_{\mathbf{2}}$ is kept secret until it is sent as $\boldsymbol{m}_{\mathbf{4}}$.

3 TTP $T$ properly processes abort requests and resolve requests in a given period.

4 The communication channels linking $U_{A}, U_{B}$ and $T$ may be insecure, i.e. attacks including eavesdropping, replaying and alteration are possible.

5 The communication channel between $U_{A}$ and $U_{B}$ may be lost permanently (i.e. either partner might escape in the middle of the exchange and the other partner is not assured of catching him).

6 The communication channel between $T$ and $U_{A}$ or $U_{B}$ may be lost, but it recovers in a given period.

7 The hash function and signatures used in the protocol are sufficiently secure; e.g. the risk of collision of the hash function or forgery of the signatures can be ignored.

8 The certificate practice is secure enough; e.g. no certificate involved in the protocol can be forged or improperly issued.

\subsection{Preventing forgery and alteration}

In the proposed protocol, forgery or alteration is possible if it is possible to store a token $v^{\prime}$ different from either $v_{1}$ or $v_{2}$ included in offer message $\boldsymbol{m}_{1}$.

There is no chance to store adifferent $v^{\prime}\left(\notin\left\{v_{1}, v_{2}\right\}\right)$ to $U_{A}$ because the offer $m_{1}$ originates from $U_{A}$ itself. $H_{A}, H_{B}$ or another faulty party may try to store $v_{i}\left(\notin\left\{v_{1}, v_{2}\right\}\right)$ to $U_{B}$ by altering $v_{1}$ or $v_{2}$ included in $m_{1}$, but this would be detected by its inconsistent hash value at step 3 in the main protocol.

Assuming $v_{1}$ in $m_{1}$ is altered to $v^{\prime}, s_{1}$ included in the $m_{2}$ (protected by signature of $\left.U_{B}\right)$ becomes $s_{1}=h\left(v^{\prime}, v_{2}, n_{1}\right)$, which is inconsistent with $h\left(v_{1}, v_{2}, n_{1}\right)$ as indicated by the comparison in step 3 of the main protocol.

The risk of forgery and alteration therefore depends on the collision resistance of hash function $h()$ and the strength of the signature, which are assumed to be sufficient.

Forgery and alteration are, therefore, prevented in the proposed protocol.

\subsection{Preventing reproduction}

In order to prevent reproduction, there must not be any token storing operation without the preceding corresponding removal operation of the same token. 
In the main protocol, any storing operation of token $v_{i}$ is performed after removing $v_{i}$. In addition, both $m_{2}$ and $m_{3}$, the evidence of the removal of $v_{2}$ and $v_{1}$, respectively, are protected against forgery or alteration by the attached signatures, and they cannot be replayed because storing $v_{1}$ and $v_{2}$ always involves the removal of $\boldsymbol{n}_{2}$ and $s_{2}$ respectively, which prevents the overlapped storing of tokens. The main protocol thus prevents the reproduction of vouchers.

A discussion about abusing the recovery protocols is more complex. We discuss the possibility of reproducing $v_{1}$ and $v_{2}$ separately.

Preventing reproduction of $\boldsymbol{v}_{\mathbf{1}} . v_{1}$ is reproduced when both of the following are performed:

$1 U_{A}$ stores $v_{1}$ in the resolve protocol.

$2 U_{B}$ stores $v_{1}$ in the main protocol or the abort protocol.

In order for $U_{A}$ to store $v_{1}$ in the resolve protocol, $U_{A}$ needs to receive abort admission $m_{a 2}$, which is signed and sent from $T$ only if $T$ has received abort request $m_{a 1}$ from $U_{B}$ before receiving resolve request $m_{r 1}$ from $U_{A}$.

Assuming $U_{B}$ sent $m_{a 1}, U_{B}$ cannot store $v_{1}$ in the main protocol because the main protocol is abandoned when $U_{B}$ starts the abort protocol. Neither can $U_{B}$ store $v_{1}$ in the abort protocol because $U_{B}$ needs to receive resolve admission $m_{a 2}$, which is sent from $T$ only if $T$ received $m_{r 1}$ before receiving $m_{a 1}$. Since this contradicts the above condition for $U_{A}$ to store $v_{1}, v_{1}$ cannot be reproduced by abusing the recovery protocols.

Preventing reproduction of $\boldsymbol{v}_{2} . \quad v_{2}$ is reproduced when both of the following are performed:

$1 U_{A}$ stores $v_{2}$ in the main protocol or the resolve protocol.

$2 U_{B}$ stores $v_{2}$ in the abort protocol.

For $U_{A}$ to store $v_{2}$ in the main protocol, $U_{A}$ needs to receive $m_{4}$, which is only known by $U_{B}$ and cannot be guessed by any other participant. If $U_{B}$ sent $m_{4}, U_{B}$ cannot store $v_{2}$ because $U_{B}$ ought to have stored $v_{1}$ and already terminated the exchange before sending $m_{\mathbf{4}}$.

If $U_{A}$ is to store $v_{2}$ in the resolve protocol, $U_{A}$ needs to receive resolve admission $m_{r 2}$, which is signed and sent from $T$ only if $T$ has not received abort request $m_{a 1}$ before receiving resolve request $m_{r 1}$. Meanwhile, in order to let $U_{B}$ store $v_{2}$ in the abort protocol, $U_{B}$ needs to receive abort admission $m_{a 2}$, which is signed and sent from $T$ only if $T$ has not received resolve request $m_{r 1}$ before receiving abort request $m_{a 1}$. Since these two conditions contradict each other, $v_{2}$ cannot be reproduced by abusing the recovery protocols. 


\subsection{Ensuring fairness}

In order to ensure fairness in voucher exchange, the following must be satisfied according to the definition of fairness described in Section 2:

1 Both $U_{A}$ and $U_{B}$ can obtain either token $v_{1}$ or $v_{2}$ (in the set $V_{A}$ and $V_{B}$ ) when the exchange is terminated.

2 Both $U_{A}$ and $U_{B}$ can terminate the exchange in finite time.

$3 v_{1}$ must not be obtained by both $U_{A}$ and $U_{B}$ simultaneously throughout the exchange; $v_{2}$ likewise.

The last condition is derived from the requirement to prevent reproduction as already discussed in Section 4.2. We therefore discuss the remaining two conditions hereafter.

In the main protocol, $U_{A}$ falls into unfair condition in which it has neither $v_{1}$ nor $v_{2}$ in $V_{A}$ while in the resolvable section, and $U_{B}$ falls into unfair condition while in the abortable section. Except in these sections, they have either $v_{1}$ or $v_{2}$ and can instantly quit the exchange at will.

While in the resolvable section, $U_{\boldsymbol{A}}$ is assured of recovering fairness and can terminate the exchange in a given period, because $U_{\boldsymbol{A}}$ may perform the resolve protocol at any time in the resolvable section, and the resolve protocol concludes in a given period provided that assumptions 3 and 6 described at the beginning of this section are satisfied. $U_{B}$ in the abortable section is also assured of recovering fairness in a similar manner.

The proposed protocol therefore guarantees fairness for both $U_{A}$ and $U_{B}$.

\section{Conclusion}

In this paper, we stated the definitions and requirements for vouchers and fairness in voucher exchanges as a basis of realizing reliable electronic commerce, and proposed a new practical protocol that enables fair and efficient (optimistic) exchanges of vouchers stored in trusted devices like smartcards, while preventing illegal acts on vouchers such as reproduction and forgery.

This protocol enables the trading participants to exchange vouchers without recourse to a TTP provided there is no accident in the exchange; it guarantees the recovery of fairness and safe termination of the exchange in a given period by accessing a TTP even if there are problems with the communication channel or dishonest acts by the partner. These properties eliminate the problems inherent in the previous method that uses a mediating TTP, including the scalability problem (traffic concentrated on the TTP) and the availability problem (TTP involved in each trade).

This protocol is thus practical and enables people participating in electronic commerce to trade with unidentified partners in complete confidence. 


\section{References}

[1] N. Asokan. Fairness in Electronic Commerce. PhD thesis, University of Waterloo, 1998.

[2] N. Asokan, V. Shoup, and M. Waidner. Asynchronous protocols for optimistic fair exchange. In Proceedings of the IEEE Symposium on Research in Security and Privacy, pages 86-99. IEEE Computer Society Press, May 1998.

[3] N. Asokan, V. Shoup, and M. Waidner. Optimistic fair exchange of digital signatures. IEEE Journal on Selected Areas in Communications, 18(4):593-610, Apr. 2000.

[4] C. Ellison and B. Schneier. Ten risks of PKI: What you're not being told about public key infrastructure. Computer Security Journal, 16(1): 1-7, 2000.

[5] K. Fujimura and D. Eastlake. RFC 3506: Requirements and Designfor Voucher Trading System (VTS), Mar. 2003.

[6] K. Fujimura and M. Terada. Trading among untrusted partners via voucher trading system. In Proceedings of the 1st Conf. on e-Commerce, e-Business, e-Government. IFIP, Oct. 2001.

[7] M. Iguchi, M. Terada, Y. Nakamura, and K. Fujimura. Voucher-integrated trading model for $\mathrm{C} 2 \mathrm{~B}$ and $\mathrm{C} 2 \mathrm{C}$ e-commerce system development. In Proceedings of the 2 nd Conf. on e-Commerce, e-Business, e-Government. IFIP, Oct. 2002.

[8] S. Kremer, O. Markowitch, and J. Zhou. An intensive survey of fair non-repudiation protocols. Computer Communications, 25(17):1606-1621, Nov. 2002.

[9] G. Lacoste, B. Pfitzmann, M. Steiner, and M. Waidner, editors. SEMPER - Secure Electronic Marketplace for Europe, volume 1854 of LNCS. Springer-Verlag, New York, NY, USA, 2000.

[10] NIST. F1PS 180-1: Secure Hash Standard, Apr. 1995.

[11] H. Pagnia, H. Vogt, and F. C.Gärtner. Fair exchange. The Computer Journal, 46( 1):55-75, Jan. 2003.

[12] M. Schunter. Optimistic Fair Exchange. PhD thesis, Universität des Saarlandes, 2000.

[13] V. Shmatikov and J. C. Mitchell. Analysis of a fair exchange protocol. In Proceedings of the 1999 FLoC Workshop on Formal Methods and Security Protocols, July 1999.

[14] M. Terada, H. Kuno, M. Hanadate, and K. Fujimura. Copy prevention scheme for rights trading infrastructure. In Proceedings of the 4th Working Conference on Smart Card Research and Advanced Applications (CARDIS), pages 51-70. IFIP, Sept. 2000.

[15] H. Vogt. Asynchronous optimistic fair exchange based on revocable items. In Proceedings of the 7th International Financial Cryptography Conference, pages 208-222. IFCA, Jan. 2003.

[16] H. Vogt, H. Pagnia, and F. C. Gärtner. Modular fair exchange protocols for electronic commerce. In Proceedings of the 15th Annual Computer Security Applications Conference, pages 3-11, Dec. 1999.

[17] J. Zhou. Achieving fair nonrepudiation in electronic transactions. Journal of Organizational Computing and Electronic Commerce, 11(4):253-267, Dec. 2001.

[18] J. Zhou, R. Deng, and F. Bao. Evolution of fair non-repudiation with TTP. In Proceedings of the 4th Australasian Conference on Information Security and Privacy (ACISP'99), pages 258-269, Apr. 1999. 